\title{
Perioperative anaesthetic challenges for intraoperative radiation therapy
}

Volume 3 Issue 5 - 2015

Keywords: intraoperative, tumor, electrocardiogram, blood pressure, pulse oximeter, capnogram, anaesthesiologists

\section{Introduction}

The anaesthesiologist's role is increasingly being approached in newer surgical therapies. The newer technique and procedures have been made possible because of development and advancement in anaesthetic practice as well. This requires the anaesthesiologists to be well versed with the nitty-gritty of the surgical procedures not only from the core surgical disciplines but also other nonsurgical streams who are performing patient centred interventions. Due to need of radiation therapy for numerous cancers either as radical treatment or adjunct treatment to other procedures, it has advanced leaps and bounce. As an adjunct treatment, radiation therapy may be administered either prior to surgical removal of the tumor or after the surgical intervention.

\section{Intraoperative radiation therapy}

Need based therapy have been supplemented with evidence based practice in the radiation oncology as well? In last few years, with the development of surgical techniques has made it possible to surgical removal of gross tumours. However, need of reducing recurrence due to residual mass remains. Thus, sophisticated techniques of irradiation are employed to reduce normal tissue margins during surgery. This technique has been labelled as Intraoperative Radiation Therapy (IORT). In IORT a concentrated dose of radiation is delivered to the tumor bed (under direct observation) exposed during the operative procedure and bed and to the tissues at risk for microscopic spread of tumor. ${ }^{1}$ This helps in destroying microscopic disease, reduces radiation treatment times with minimum affect on the normal organs..$^{1,2}$ In IORT targeted boost is provided to the affected area with accurate localisation and minimum exposure to the normal area. This method of treatment has been used to treat locally advanced malignant lesions when conventional modalities may not produce local tumor control like in colorectal cancer, retroperitoneal sarcomas, limb sarcomas, gynaecologic malignancies, and paediatric malignancies. ${ }^{3-5}$ Patient selection for IORT is important and patients with localised tumor, (no distant metastasis) not expected to be cured by surgical intervention alone with no contraindications to surgery and anaesthesia are considered for IORT. ${ }^{1,2}$

\section{IORT and anaesthesiologists}

The technique of IORT has some challenges and prerequisites. ${ }^{6-9}$ This pertains to site of the treatment, patient related factors and surgery related factors. During the IORT, patient requires to be still so as to prevent damage to the nearby tissues and delivery of maximum dose to the targeted area at the tumour bed and the marginal area. ${ }^{10,11}$ Conventionally for radiation therapy in adults, general anaesthesia is not required and patient is advised to remain still during the radiation therapy. However for IORT, since the surgical intervention is ongoing, patient cooperation is not feasible. This means that these therapies

\author{
Nishkarsh Gupta,' Anju Gupta, ${ }^{2}$ Rakesh \\ Garg' \\ 'Department of Anaesthesiology, Pain and Palliative Care, India \\ ${ }^{2}$ Department of Anaesthesiology, Maulana Azad Medical College, \\ India
}

Correspondence: Rakesh Garg, Department of

Anaesthesiology, Pain and Palliative Care, Dr BRAIRCH, Room No. 139, I st floor, All India Institute of Medical Sciences, Ansari Nagar, New Delhi-I I 0029, India, Tel 91 9810394950; +91 9868398335, Email drrgarg@hotmail.com

Received: July 02, 2015 | Published: December 21, 2015

may not be effective if the patient cannot stay still with minimum movement for the required time.

\section{Anaesthetic management}

Anaesthesiologists play an important role in intraoperative irradiation. It requires pre-procedure assessment not only for surgical intervention but also for radiation therapy. Assessment needs to be focussed on acute effects of radiation in addition to surgical insult. In the preoperative period the patients must be thoroughly evaluated for any coexisting systemic disease and side effects of chemotherapy and external radiation treatments. In the operating room standard monitors including electrocardiogram, blood pressure, pulse oximeter, capnogram and temperature monitoring should be used depending upon the patient's condition and the surgical procedure planned. Other monitoring like invasive pressure monitoring, urine output, cardic output depends on associated co-morbidities and complexity of the surgical procedure.

It is advisable to give general anaesthesia to these patients because the position and movements may be uncomfortable and cause anxiety to the patients and also even slightest of movement during the treatment may affect the outcome. No anaesthetic drug is ideal and the anaesthetic induction drugs, inhalation agents, muscle relaxants and opioids will vary depending upon the anaesthesiologist preference, patient's requirement and availability and intervention planned. Although concrete evidence is not available, it appears that general anaesthetic regimen with endotracheal intubation is acceptable choice. Total intravenous anaesthesia (TIVA) with propofol and remifentanyl may also be considered for IORT. For IORT additional anaesthesia time (30-45 minutes) is required for placing the linear accelerator on the tumor bed and treatment time.

The place of IORT varies as per infrastructure. The option available includes:

a. Dedicated IORT suite for anaesthesia, surgical incision, tumor isolation and radiation delivery occur in dedicated IORT OT. In 
this the radiation is given by mobile radiotherapy system (like INTRABEAM ${ }^{\circledR}$ Carl Zeiss Meditec, Germany) using shortrange X-ray radiation. After induction of anaesthesia, surgery is accomplished and thereafter the OT table is repositioned to target radiation beam to give IORT. After the therapy the OT table is returned to the original position.

b. There is a standalone operation theatre in the radiation oncology area. After anaesthesia, tumor is isolated and thereafter the patient is shifted to the accelerator room. After radiation treatment the patient is shifted back to the OT in the radiation area for closure of the incision. It is potentially difficult situation and a mishap can easily occur during the transfer. This kind of setup has limitations similar to procedures requiring anaesthesia outside the operating room. ${ }^{12}$

In both these technique the anaesthetic maintenance continues during the short exposure to the radiation (approximately 30 minutes). During these hours routine monitoring as per standard needs to be continued. However, all the theatre staff including the anaesthesiologist leaves the operating room when the radiation therapy is going on. So for a brief period the patient has to be left unattended and is supervised through a lead glass window by the anaesthesiologist. A slave monitor is also attached for monitoring the vitals outside the radiation area. This can be really challenging and stressful as anaesthesiologists have to control the vitals on basis of a set of monitors from a distance. There facility should have arrangements to monitor the patient's vitals from outside the operating area and allow instant physical access to the patient in case of emergency.

The setup for IORT needs to be cautiously prepared from the beginning itself. The following things should be present for remote monitoring:

a. Glass window to monitor the patient

b. A monitor connected to anaesthesia machine to relay beat to beat vitals of the patient

c. A loudspeaker connected to the cable of microphone in the esophageal stethoscope can help the anaesthesiologist to hear both heart and breath sounds during treatment. This may help in early detection of apnoea from disconnected breathing circuit.

d. Wall mounted cameras in treatment room connected to television so as to monitor the patient continuously during the procedure. The camera should be zoomed to have clear view of anaesthesia machine (flow meter, ventilator bellows, digital display of vitals) and other should focus on the patient to identify any patient movement (mean duration 2.5- 20 minutes). There should be a system to stop the acceleration in case the door is open so that anaesthesiologist can reach the patient in the shortest possible time.

e. After treatment is complete, anaesthesia is reversed and patient is shifted to recovery room for observation. In case of emergency the treatment is stopped to allow direct access to the patient.

\section{Conclusion}

The anaesthesia for IORT is challenging for anaesthesiologist. Proper planning of the setup, anticipation of the problems and coordination with surgical and radiation colleagues is important for uneventful management.

\section{Acknowledgments}

None.

\section{Conflicts of Interset}

None.

\section{References}

1. Gunderson LL. Rationale for and results of intraoperative radiation therapy. Cancer. 1994;74(2):537-541.

2. Arain MR, Buggy DJ. Anaesthesia for cancer patients. Curr Opin Anaesth. 2007;20(3):247-253.

3. Petersen IA, Haddock MG, Donahue JH, et al. Use of intraoperative electron beam radiotherapy in the management of retroperitoneal soft tissue sarcomas. Int J Radiat Oncol Biol Phys. 2002;52(2):469-475.

4. Taylor WE, Donohue JH, Gunderson LL, et al. The Mayo Clinic experience with multimodality treatment of locally advanced or recurrent colon cancer. Ann Surg Oncol. 2002;9(2):177-185.

5. Haddock MG, Petersen IA, Webb MJ. IORT for locally advanced gynecologic malignancies. Front Radiat Ther Oncol. 1997;31:256-259.

6. Ritchey ML, Gunderson LL, Smithson WA, et al. Pediatric urological complications with intraoperative radiation therapy. $J$ Urol. 1990;143(1):89-91.

7. Tepper JE, Gunderson LL, Orlow E, et al. Complications of intraoperative radiation therapy. Int J Radiat Oncol Biol Phys. 1984;10(10):1831-1839.

8. Haddock MG, Petersen IA, Pritchard D, et al. IORT in the management of extremity and limb girdle soft tissue sarcomas. Front Radiat Ther Oncol. 1987;31:151-152.

9. Schomberg PJ, Gunderson LL, Moir CR, et al. Intraoperative electron irradiation in the management of pediatric malignancies. Cancer. 1987;79(11):2251-2256.

10. Wolff BG, Gunderson LL. Intraoperative radiation therapy for rectal carcinoma. Semin Colon Rectal Surg. 1980;1:44- 47.

11. Gunderson LL, Martin JK, Earle JD, et al. Intraoperative and external beam irradiation with or without resection: Mayo pilot experience. Mayo Clin Proc. 1984;59(10):691-699.

12. Frankel A. Patient safety: anesthesia in remote locations. Anesthesiol Clin. 2009;27(1):127-139. 